4. That whenever the slightest probability of tions and are often ignorant that they have any pregnancy exists, it should be fully explained to disease. On the other hand, we, with equal frethe patient and her friends.

5. That the necessity for operative relief and the consequences of delay or neglect should be carefully stated to the parties interested, before obtaining their formal consent to the operation. 列 port fully all such cases, that the methods of way that we are most likely also to determine a diagnosis may be improved, if possible.

7. That it is the duty of the profession at large to maintain that pregnancy may be absolutely concealed, especially prior to the fourth or fifth month, by other intra-abdominal conditions.

Bibliography in addition to references found in tables :

Barnes, Simpson, Hart and Barbour, Hewitt, Jones, Courty, Scanzona, Hagar and Kaltenbach, Pean, Hofmeier, von Flammerdingle, Tait, Wells, Thomas, Emmett, Skene, Byford and Goodell.

Obstetrics-Barnes, Playfair, Simpson, Leichman, Schroeder, Speigelberg, Cazeaux and Tarnier, Lusk.

Reports-."London and Edinburgh Obstetrical," "St. George and Guy's Hospital Report," "Journal British Gynæcological Society," "American Journal of Obstetrics," "Annals Gynæcology," "Transactions of the American Gynæcological Society," etc.

rational treatment for the disease.

It seems evident that nearly all the most important symptoms of chronic endocarditis are due to the disturbances produced in the distribution of the blood throughout the body. It is by these disturbances of the circulation that the cerebral and pulmonary symptoms, the loss of nutrition and the dropsy are produced. The problem before us, therefore, is to determine why in some cases of chronic endocarditis there are disturbances of the circulation, and why in other cases there are not.

It might seem at first that the solution of this problem is an easy one, that the disturbances in the circulation are simply in proportion to the stenosis or insufficiency of the valves. A very moderate experience, however, is sufficient to show that this is not the case. The problem is a complicated one, and the disturbances of the circulation are due to a number of causes which act singly or together.

We may enumerate these causes as follows:

The endocarditis.

\title{
CHRONIC ENDOCARDITIS.
}

Read in the Section of Practice of Medicine at the Foriteth Annual Meting of the American Medical Association. Itne 28, 1880.

BY FRANCIS DELAFIELD, M.D., OF NEW YORK.

It is proper that I should offer to you some reasons for selecting so ordinary a disease as is chronic endocarditis as the subject for this paper. But the ordinary diseases are, after all the important ones, and often as we see them we never really know them well enough. Any fresh record of facts, any new way of grouping, or of looking at these facts nust be of some little service. It is the object of this paper, therefore, not to give a systematic account of endocarditis, but to draw attention to some features of the disease; not to give a history of the views of other observers, but to state simply what I have put together from my case books and post-mortem records.

Of the ordinary diseases few are more common than is chronic endocarditis. In few of them is there so great a variety in the severity of the symptoms. From the condition of a trifling inconvenience to that of a distressing and fatal disease there seems to be no limit to its various phases. We constantly meet with patients whose heart valves are seriously damaged and who yet enjoy good health, can follow laborious occupa-

The dilatation and hypertrophy of the ventricles.

The inflammation or degeneration of the wall of the heart.

The inflammation of the coronary arteries.

The abnormal heart action.

The associated pulmonary emphysema, chronic endarteritis, and chronic Bright's disease.

To follow out the mode of action of all these causes is not possible in a paper of this character. I confine myself to the consideration of three of them: The endocarditis; the abnormal heart action; and the secondary and complicating changes in the kidneys. Not that the others are unimportant, but that these three are perhaps the most important of all.

I. The Endocarditis.

In thinking of persons with valvular lesions, we must remember that some of these persons, while under our observation, are suffering from chronic endocarditis, and that some are only suffering from the changes produced in the valve by an endocarditis which no longer extsts. In the one case they suffer from a chronic inflammation, in the other from a deformity. It may be indeed that such deformity leads to progressive changes in the cavities and walls of the heart. But this is much less likely to happen than if the changes in the valves are also progressive. 
Chronic endocarditis may directly follow acute endocarditis; it may be developed after a long interval in valves damaged by an old attack of endocarditis; it may from the first be a chronic lesion. We find, especially in children, an uninterrupted history of cardiac symptoms beginning with an acute attack and continuing as a chronic disease for years. We find in older persons with marked cardiac symptoms developed late in life a history of an acute endocarditis in childhood, from which they had apparently recovered. We find in adults the gradual development of one cardiac symptom after another, so slow and so gradual that we can hardly date the beginning of the disease.

Chronic endocarditis, when it has once commenced, seems to have a natural tendency to persist and to involve other portions of the endocardium. The cases vary as to the activity of the endocarditis and the intermissions in the course of the inflammation. It is apparently possible for the endocarditis to stop at any time, and the valves will then undergo no further change. Chronic endocarditis is a productive inflammation with the formation of new tissue, but without exudation. In its most active form there is a very considerable growth of cells and also a death of cells, so that the inflamed endocardium is thick ened in some places, ulcerated in other, and on the roughened surfaces thus made, thrombi are formed. This is the most active and dangerous form of the disease. In its more chronic form the growth of cells is not as great, the cells do not die, the basement substance is thickened. Although the surface of the endocardium is somewhat roughened, and small vegetations are often formed, there are no thrombi.

In either form of inflammation there may be added degeneration or calcification of the inflamed endocardium and of the thronbi. It may very well happen that the patient, after suffering from the chronic form of endocarditis for years, may then develop the more active form in the same valve, or in one of the other valves.

In the more active form of the disease a large number of the cases run their course within six months from the time of the commencement of their symptoms. A considerable number do not live longer than six or seven weeks. The symp toms are pronounced: Disturbed heart action; delirium, convulsions, paraplegia ; cough, hæmop. tysis, dyspnca; nausea and vomiting; dropsy; loss of flesh and strength and anæmia, and a rise of temperature. These patients are apt to get rapidly worse, but there may be intermissions, and the inflammation may stop aitogether. It seems evident that in the treatment of these patients we must remember that they are suffering from an inflammation of some activity, and that rest in bed, the use of cold or of counter irritation over the heart are measures likely to be of use.
On the other hand, in the slow form of endocarditis the disease lasts for many years. There are usually intermission in its course, and it may stop altogether at any times. Many of the patients have no symptoms. In those who do, some one symptom is first developed and then, as the disease progresses, others are added. These patients are regularly better for an out of door life, with as much exercise as they are able to take.

2.-The Abnormal Heart Action.

It is possible for a chronic endocarditis to run its entire course with a perfectly regular action of the heart. This, however, is the exception. The rule is, that the heart's action is disturbed, and this disturbance is often the most important feature of the disease, and furnishes the principal indication for treatment. Such disturbance of the heart's action may be due to:

a. The endocarditis existing as an inflammation of some activity and producing changes in the heart's action in the same way as does an acute endocarditis.

b. Such a degree of stenosis, or insufficiency of the valves as will mechanically interfere with the heart's action. This often does not become a factor of much importance until the stenosis or insufficiency are well marked, and for this reason it is easy to be deceived as to the character of the lesion.

c. Dilatation and hypertrophy of the ventricles are often present, and certainly have their effect in changing the character of the heart's action.

d. Chronic myocarditis and disease of the coronary arteries produce the most extreme and intractable disturbances of the heart's action. Fortunately they are not very common.

e. Contraction of the smaller arteries throughout the body with increase of arterial tension and venous congestion. This condition, although a frequent complication of endocarditis, nephritis, endarteritis, emphysema, and occurring as an in. dependent condition in some cases of angina pectoris, is yet something concerning which our knowledge is imperfect. Whether contaminated blood irritates the arteries, whether the nervous centers are irritated by the contaminated blood or in what way the contraction of the arteries is produced, we do not know. But such a contraction is produced and lasts for hours, days or months. The same patient may never have but one such attack, or he may have many. In patients who have had many attacks, the muscular coat of the small arteries is thickened. Such a contraction of the arteries at once changes the character of the heart's action. It becomes rapid, forcible, feeble and tumultuous. The degree of the cardiac disturbance is apparently in proportion to the degree and suddenness of the contraction of the arteries, but is not related to the severity or extent of the endocarditis. In the early stages of endocarditis we very often see patients who 
complain of dyspnoea on exertion and on lying down with precoraial pain. Otherwise they feel perfectly well and have normal urine. We find the heart enlarged, its action rapid and forcible, and a murmur indicating disease of one of the valves. The radial pulse is distinctly tense. If by treatment the arteries are dilated the dyspnoea disappears, the heart's action becomes natural and the patients feel well. Or, in a further advanced and progressing endocarditis we may follow patients for years who, in spite of their endocarditis, feel well except when they have attacks of contraction of the arteries. When they have such an attack dyspncea and other symptoms are developed and continue for weeks or months. Then as the attack subsides, the symptoms disappear and the patients feel well. The first attacks yield readily to treatment. But as time goes on the attacks are more frequent and more obstinate. The pulse is tense, but small. The heart's action is no longer forcible, but feeble or tumultuous. Occasionally we see patients who go on with a chronic endocarditis for many years, but with few or no symptoms. Then with a slight pleurisy, or pericarditis, or without discoverable cause a sudden, extreme and intractable contraction of the arteries is established, causing the most urgent dyspnoea and continuing up to the time of the patient's death. These attacks of the contraction of the arteries are at first readily relieved by the drugs which dilate the arteries-nitrate of amyl, chloral hydrate, nitro-glycerine, opium and potassium iodide. The patients do well with an out-of-door life and regulated exercise. But as the endocarditis advances and the valves are more damaged, especially by stenosis, these attacks are less readily relieved, and it becomes necessary to keep the patients more and more quiet.

f. Unknown causes which apparentiy act through the nerves which regulate the action of the heart. These form a large, important and obscure group of cases. In some of these cases the abnormal heart action is associated with advanced disease of the valves, and it is only by the results of treatment that we can discriminate how much of the disturbance of the heart's action is due to the valvular lesion, and how much to nervous influences. The pulse is of low tension, feeble and rapid. The heart's action is feeble or exaggerated. The condition of the patients is often very bad, and yet in some of them very marked improvement is obtained by treatment.

These patients at first require complete rest, then massage and later regulated exercise. The most efficient drugs are digitalis, strophanthus, caffein, convallaria and barium chloride. The heart seems to be always really a weak heart even though its action is exaggerated. It is often natural to believe that there is degeneration or inflammation of the walls of the ventricles, but yet after death no such changes are found. In other cases the endocarditis is not advanced, the valves are but slightly narrowed or insufficient, there is little or no change in the size of the heart. The heart's action is rapid, either feeble or exaggerated. The pulse is soft and rapid. The patients often have pain or abnormal sensations referred to the heart, the general health may be very much impaired. Although these patients have endocarditis, yet it is really the abnormal heart action which makes them ill and calls for treatment. Some of these patients are very easily managed, the heart's action soon becomes normal, the pain disappears and the patients feel well, although the lesion of the valve still exists. Some of them, on the contrary, do not improve. The disturbance of the heart's action and other symptoms continue, but yet the patients do not die, nor get worse beyond a certain point. There are, however, occasional cases in which the heart's action becomes very bad, the patients are very feeble and die. After death we find but moderate changes in the valves and no changes in the walls of the heart. The management of these cases is apt to be difficult. Attention to the diet, the general health, the habits, the climate, the exercise is of especial importance. Of drugs, one or other of the cardiac stimulants is often indicated.

3. The secondary and complicating changes in the kidneys.

Of the persons who suffer from chronic endocarditis a large number never have any complicating disease of the kidneys. In the persons who die from endocarditis it is rare to find normal kidneys. In judging of the frequency of the kidney lesions the most certain criterion is the autopsy. During life it is not sufficient to examine for albumen and casts, which are often absent, but the quantity of the urine, its specific gravity, and the proportion of urea to the ounce of urine must also be determined. Advanced changes in the kidneys often exist in patients whose urine is said, after a superficial examination, to be normal.

In persons who die from chronic endocarditis we find :

Chronic congestion of the kidney.

Chronic degeneration of the kidney.

Chronic nephritis.

I. Chronic congestion of the kidney.

The kidneys are of medium size, or large. Their weight is increased, they are hard, uniformly congested, their surfaces are smooth. The epithelium of the cortex tubes is opaque, flattened or swollen. The glomeruli show a dilatation of the capillaries, with more or less thickening of their walls and the swelling of the cells which cover their walls. In the stroma there is nothing but some exaggeration of the subcapsular areas of connective tissue which are found in 
normal kidneys. The arteries are normal, the is opaque, swollen or flattened. The tubes may pyramid veins are congested and sometimes di-contain coagulated matter. The straight tubes lated. The urine is diminished in quantity at of the cortex and pyramids may contain cast matthe times when the heart's action is bad, and re ter. The capillaries of the glomeruli are swollen, turns to the normal when it is better. It is ap- their walls are thickened, there is an increase in parently never increased except from accidental the size and number of the cells which cover the causes. The specific gravity is usually between capillaries. There is a considerable growth of I020 and 1025, but may for a time be down to Ioro, or up to 1035. The quantity of urea is rarely less than Io grains to the ounce, it may be as high as $2 \mathrm{I}$ grains. The specific gravity and the quantity of urea must be judged by examining the urine of 24 hours for several days, with the proper allowance for diet and exercise. Albumen and casts are absent or present in very small quantities. The effect of the congestion of the kidneys on their functions is simply to diminish the quantity of urine. The quality of the urine is good, and the exudation from the vessels amounts practically to nothing. Apparently the only way in which this lesion of the kidney can add to the symptoms of the endocarditis, is by the diminution in the quantity of the urine.

2. Chronic degeneration of the kidney.

The kidneys are considerably increased in size and weight, weighing together from $\mathrm{I} 6$ to 20 ounces. Their surfaces are smooth; the cortical portion is thickened, of pink or white color, the pyramids are red. The gross appearance is that of the so-called large white kidney. The epithelium of the cortex tubes is swollen and opaque. In the glomeruli there is dilation of the capillaries. There are no changes in the stroma, or in the arteries, the pyramid veins may be congested. The quantity of the urine varies with the changes in the action of the heart, sometime abundant, sometimes scanty, sometimes suppressed. The specific gravity is not diminished, nor is the proportion of urea to the ounce decreased. Albumen and casts in small quantities are more frequently present than with chronic congestion. While it is difficult to separate the kidney symptoms from the heart symptoms, yet one has the impression that this kidney lesion is more serious than chronic congestion, and has its effect in increasing the symptoms of the endocarditis, especially the loss of nutrition and the anæmia.

3. Chronic nephritis,

A chronic inflammation of the kidneys may follow chronic congestion or chronic degeneration, it is then evidently a direct result of the endocarditis ; or it may be developed as an independent inflammation and is to be regarded as an as sociated and not a secondary lesion.

a. Chronic nephritis following chronic congestion of the kidney. The kidneys remain increased in size, or become somewhat smaller. The capsules are adherent, the surface of the kidney is finally nodular, the consistence of the organ remains hard, and the general venous congestion continues. The epithelium of the cortex tubes The quantity of exudation from the blood vessels 
and the rapidity of the changes in the kidney. To these two points of difference correspond the differences in the clinical histories. We may, therefore, for clinical purposes distinguish three forms of chronic nephritis.

1. Chronic nephritis with large and continued exudation of serum from the blood vessels of the kidney into the tubes. The urine is sometimes diminished, sometimes very much increased in quantity. The specific gravity is lowered, often at about ror6. The proportion of urea to the ounce of urine is diminished. The urine constantly contains considerable quantities of albumen and numbers of casts. The patients do badly. Dropsy, anæmia, loss of flesh and strength and chronic uræmia are the regular symptoms.

2. Chronic nephritis with moderate and intermittent exudation from the blood vessels. The urine is more or less increased in quantity, except when the patient has an attack of contraction of the arteries, then it is diminished. The specific gravity is lowered. The proportion of urea to the ounce of urine is diminished. During much of the time no albumen or casts are present, but from time to time when the patient is doing badly in other ways they appear in moderate quantities. These patients usually live for a num. ber of years, slowly getting worse. They are especially liable to attacks of contraction of the arteries with dyspnœea, headache, sleeplessness and convulsions.

3. Chronic nephritis with little or no exudation from the blood vessels, the nephritis advancing very slowly. These kidneys are considered by some authors to be examples of fibroid degeneration, rather than of chronic inflammation. The urine shows no change except that the specific gravity and the proportion of urea from year to year are gradually lowered. Some of the patients never have any renal symptoms. Some of them have attacks of contraction of the arteries. Some of them simply lose some flesh and a great deal of strength and die quietly

\section{PROPERITONEAL HERNIA.}

Read in the Section of Surgery and Anatomy at the Fortieth An unal Meeting of the American Medical Association, held at Neziport, June, r880.

BY CHARLES W. DULLES, M.D.,

SURGEON TO OUT-PATIENTS IN THE HOSPITAL OF THE UNIVERSITY

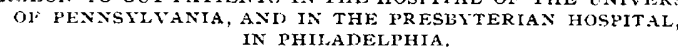

The subject of properitoneal hernia is so little known in this country that I believe that there have been but three cases with this title recorded by American surgeons. One of these I reported to the Philadelphia Academy of Surgery, December 6, I 886; the second was reported by Dr. Hartley, of New York, in the N. Y. Medical Record, about a week later; and the third by Dr. Torrey, of Brooklyn, in the Annals of Surgery, March, I 888 .

For this reason I have thought it worth while to bring the subject before this body for consideration and discussion.

In studying the literature of properitoneal hernia I have come to the conclusion that very many cases must have come under the observation of American surgeons who did not classify them as they would have done had they understood them thoroughly. One reason for this conviction rests upon the titles which are at the head of a number of articles upon hernia, as they may be found recorded in the Index Catalogue of the SurgeonGeneral's Library at Washington. Another rests upon the fact that since the careful studies of this subject by Streubel, Krönlein and Küster in Germany have been published, a considerable number of cases have been specifically recorded in that and in other countries.

It must not be supposed that this fact indicates a mere refinement in diagnosis. It means much more than this; for, before these studies were made, the history of properitoneal hernia was one of unvaried disaster. The diagnosis was, we may say, invariably made on the post-mortem table. Since Krönlein pressed his opinions upon the attention of his professional brethren the state of affairs has been very different. Now this form of hernia can not only be diagnosticated during the life of the patient, but it can also be successfully treated.

In a general way a properitoneal hernia may be said to be one which occupies an abnormal position within the abdoninal or pelvic wall in front of the peritoneum. Sonnenburg has suggested that the term præperitoneal would be more exact. This is true; but we may, I think, hold to the term proposed by Krönlein, lest we introduce confusion by a change which is not important.

A true properitoneal hernia lies between the parietal peritoneum and the overlying mass of muscles, or-when pelvic-the bones. There is also a form of hernia which occupies a position between the planes of the abdominal muscles, which may be called "intermuscular;" and another which lies outside of them and just beneath the skin and superficial fascia. For the last Küster has proposed the term " hernia inguino-superficialis," and he divides it into three varieties: I, hernia inguino-superficialis abdominalis ; 2 , hernia inguino-superficialis cruralis; and 3 , hernia inguino-superficialis perinealis; as they occupy respectively the region of the lower abdominal wall, of the thigh, or of the perineum.

The three principal forms of hernia which escape from the usual route of the inguinal canal and the scrotum are: properitoneal, intermuscular and superficial hernia.

I have now collected and studied the histories of thirty-four cases of properitoneal hernia. In 